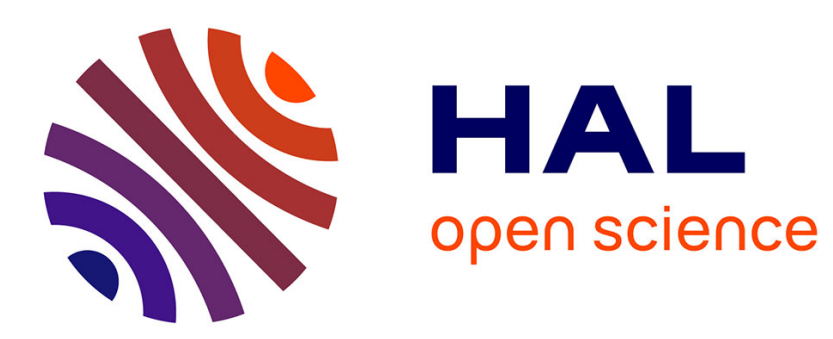

\title{
Online adaptation of reference trajectories for the control of walking systems
}

Pierre-Brice Wieber, Christine Chevallereau

\section{To cite this version:}

Pierre-Brice Wieber, Christine Chevallereau. Online adaptation of reference trajectories for the control of walking systems. Robotics and Autonomous Systems, 2006, 54 (7), pp.559-566. 10.1016/j.robot.2006.04.007 . inria-00390423

\section{HAL Id: inria-00390423 https://hal.inria.fr/inria-00390423}

Submitted on 2 Jun 2009

HAL is a multi-disciplinary open access archive for the deposit and dissemination of scientific research documents, whether they are published or not. The documents may come from teaching and research institutions in France or abroad, or from public or private research centers.
L'archive ouverte pluridisciplinaire HAL, est destinée au dépôt et à la diffusion de documents scientifiques de niveau recherche, publiés ou non, émanant des établissements d'enseignement et de recherche français ou étrangers, des laboratoires publics ou privés. 


\title{
Online adaptation of reference trajectories for the control of walking systems
}

\author{
Pierre-Brice Wieber ${ }^{\mathrm{a}}{ }^{*}$ Christine Chevallereau ${ }^{\mathrm{b}}$ \\ ${ }^{a}$ INRIA Rhône-Alpes, Zirst Montbonnot, 38334 St Ismier Cedex - France \\ ${ }^{\mathrm{b}}$ IRCCyN, 1 rue de la Nö̈, BP 92101, 44321 Nantes Cedex 03 - France
}

\begin{abstract}
A simple and widely used way to make a robotic system walk without falling is to make it track a reference trajectory in one way or another, but the stability obtained this way may be limited and even small perturbations may lead to a fall. We propose here a series of heuristics to improve the stability that can be obtained from such a tracking control law, through an online adaptation of the choice of the reference trajectory being tracked. Encouraging simulations are obtained in the end on a simple planar biped model.
\end{abstract}

Key words: Online adaptation; parametrized trajectories; constrained dynamics; walking systems; viability

\section{Introduction}

When a mechanical system is walking, it has possibly many contacts with the ground which are regularly broken and recovered in order to produce a displacement of the whole structure. This allows to travel across obstacles with great versatility, but at the cost of a strong instability stemming from the fact that the dynamics of walking systems depends strongly on the forces that can be obtained from these contacts.

Being concerned with the stability of walking systems, this publication begins therefore in section 2 with a general model of their dynamics that builds on a unilateral model of the contacts with the ground and allows to specify which

\footnotetext{
* Corresponding author.

Email addresses: Pierre-Brice.Wieber@inrialpes.fr (Pierre-Brice Wieber), Christine.Chevallereau@irccyn.ec-nantes.fr (Christine Chevallereau).
} 
movements a walking system can do and which movements it can't $[13,14]$. The possibility to avoid to fall can be modeled then as a viability and invariance property.

A simple and widely used way to obtain such invariance properties is to make a walking system track a reference trajectory in one way or another [2-4,6-15], but the invariance obtained this way may be limited and even small perturbations may lead to a fall. How to improve the capacity for a walking system to avoid to fall has therefore been the main goal of most of the researches done in the field of walking systems, and especially in the field of biped walking systems since this stability issue is particularly problematic for them.

Since the problem is the availability of contact forces, it has been proposed to deal more properly with them by lowering the needs of the trajectory tracking, tracking for example trajectories with only some parts of the system $[10,12]$ or allowing some deviations from the reference trajectories when forced to do so $[4,6,10]$. It has been proposed also to adapt the reference trajectory being tracked to the availability of contact forces, but most of such propositions so far don't clearly define when and how such an adaptation should occur, relying on parameters that need to set with no clear relation to the global stability of the system $[8,15]$. A more radical approach is even to completely generate online the reference trajectories [2], but in a way, all of these approaches blur the effects of tracking reference trajectories, leading to an uncertain result as to really improving the capacity to avoid to fall.

Building on the analysis of section 2, we propose here, in section 3, a series of heuristics for the online adaptation of the reference trajectory being tracked which builds on a well delimited set of reference trajectories and a strict tracking which continuously keeps an eye on the available forces [14]. We show then in section 4 how to apply these heuristics to a simple planar biped model, leading to encouraging numerical experiments in section 5 .

\section{The dynamics and stability of walking systems}

\subsection{Structure of the dynamics}

Whatever the walking system being considered, planar or three-dimensional, with any number of legs with or without feet, its dynamics can be classically written as a set of Euler-Lagrange equations:

$$
M(q) \ddot{q}+N(q, \dot{q}) \dot{q}+G(q)=T(q) u+C(q)^{T} \lambda
$$


where $T(q) u$ are actuation forces and $C(q)^{T} \lambda$ contact forces.

As for any mechanical system that is able to move around, its configuration vector $q$ has to account for two different informations, the shape of the system on one side, its position and orientation in space on the other $[6,11,13]$. The shape of the system can be described by its joint positions, a vector $q_{1}$, and its position and orientation in space can be described by the position and orientation of a frame attached to one of its parts, leading to a vector $q_{2}$ of dimension 3 for planar systems, 6 for three-dimensional systems.

If we consider then the structure of the vector $q$ :

$$
q=\left[\begin{array}{l}
q_{1} \\
q_{2}
\end{array}\right]
$$

we can split the dynamics (1) to exhibit the same structure:

$$
\left[\begin{array}{c}
M_{1}(q) \\
M_{2}(q)
\end{array}\right] \ddot{q}+\left[\begin{array}{c}
N_{1}(q, \dot{q}) \\
N_{2}(q, \dot{q})
\end{array}\right] \dot{q}+\left[\begin{array}{l}
G_{1}(q) \\
G_{2}(q)
\end{array}\right]=\left[\begin{array}{c}
T_{1}(q) \\
0
\end{array}\right] u+\left[\begin{array}{l}
C_{1}(q)^{T} \\
C_{2}(q)^{T}
\end{array}\right] \lambda
$$

where the actuation forces don't appear in the lower part $[6,11,13]$ :

$$
M_{2}(q) \ddot{q}+N_{2}(q, \dot{q}) \dot{q}+G_{2}(q)=C_{2}(q)^{T} \lambda
$$

\subsection{Contact forces}

It appears then that for a walking system to realize a movement $q(t)$, equation (3) must be satisfied with appropriate contact forces. But the physics of contact is such that these forces have limitations: in the general case (no gluing, especially), contacting solids can push one another but they can't pull one another (what is referred to as the unilaterality of contacts), and friction between them is limited $[6,7,9,13]$. This can be expressed as a vector inequality on the amplitudes $\lambda$ of the contact forces:

$$
\mathcal{A}(\lambda) \leq 0
$$

Considering this restriction of contact forces together with the lower part of the dynamics (3), a necessary condition for a walking system to realize a 
movement $q(t)$ is that there exist contact forces $\lambda(t)$ such that:

$$
\left\{\begin{array}{l}
M_{2}(q) \ddot{q}+N_{2}(q, \dot{q}) \dot{q}+G_{2}(q)=C_{2}(q)^{T} \lambda \\
\mathcal{A}(\lambda) \leq 0
\end{array}\right.
$$

Note that this condition can be shown to be a complete generalization of more usual criteria such as the Center of Pressure or the Zero Moment Point criteria [14].

\subsection{Impacts}

Note also that when a part of a walking system lands on the ground, a sharp change of velocity may happen, an impact which can be modeled as an instantaneous event, especially in the case of purely rigid bodies $[9,13]$. This way, an instantaneous version of the Euler-Lagrange equations:

$$
M(q)\left[\dot{q}_{+}-\dot{q}_{-}\right]=C(q)^{T} \Lambda
$$

relates the velocity of the system before and after the impact, $\dot{q}_{-}$and $\dot{q}_{+}$, to impulsive contact forces $C(q)^{T} \Lambda$.

\subsection{Avoiding to fall, a viability condition}

Condition (5) shows that a walking system's ability to control its movements, and especially to keep its balance, is bound to the availability of appropriate contact forces: falling is a permanent threat then, and a threat for the integrity of both the walking system and its environment. Avoiding to fall should therefore be considered as an essential condition for walking systems, to be taken care of before any other goal.

Now, if we consider the set $\mathcal{F}$ of positions where the system is considered as having fallen (where a part of the system other than the feet is in contact with the ground, for example), avoiding to fall means avoiding to be in a position $q \in \mathcal{F}$. A viability condition $[1,13]$ naturally comes out then:

Definition : A state $(q, \dot{q})$ is considered as viable if and only if the system is able to realize a movement $q(t)$ starting from this state that never gets inside the set $\mathcal{F}$. 


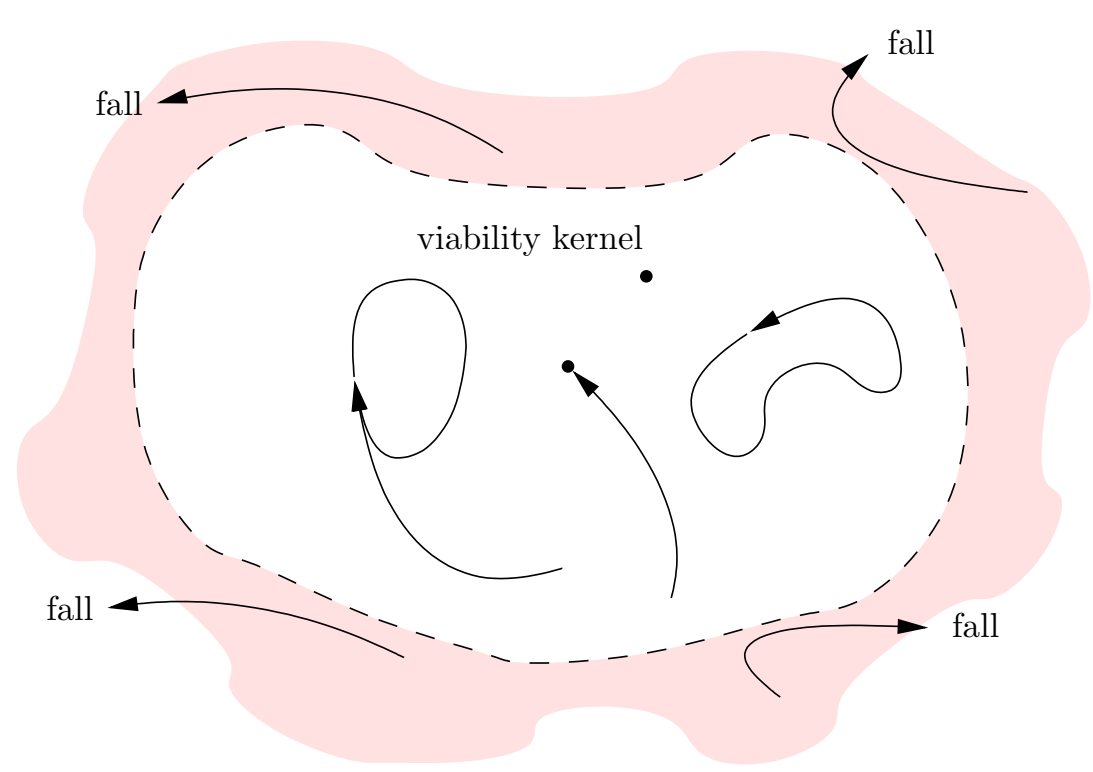

Fig. 1. The viability kernel gathers all the states from which it is possible to avoid to fall, equilibrium points and cyclic movements among others. The states outside this kernel are those from which a fall cannot be avoided.

A state $(q, \dot{q})$ is therefore either viable and the system is able to avoid to fall from it, or non-viable and the system cannot avoid to fall from it. This way, if we consider the viability kernel, union of all viable states (figure 1), avoiding to fall means always staying inside this kernel, what should be considered then as the primary goal for every control law of walking systems.

This concept of viability appears to be very general: it applies to any type of locomotion, any structure of robot as long as falling is considered as an event that should be avoided. It is unfortunately of poor practical use since the complexity of the dynamics of walking systems is such that it is generally computationally impossible to verify whether a state is viable or not.

Numerous viable states can be sorted out though, equilibrium points, cyclic movements or trajectories leading to one of these, so that viability still seems to be an interesting concept to refer to in the analysis and design of control laws, as we will see in the next section.

\section{Tracking reference trajectories}

\subsection{Adapting the choice of the reference trajectories}

A simple and widely used way to make a walking system avoid to fall is to make it track a reference trajectory, in one way or another [2-4,6-15]. Indeed, 

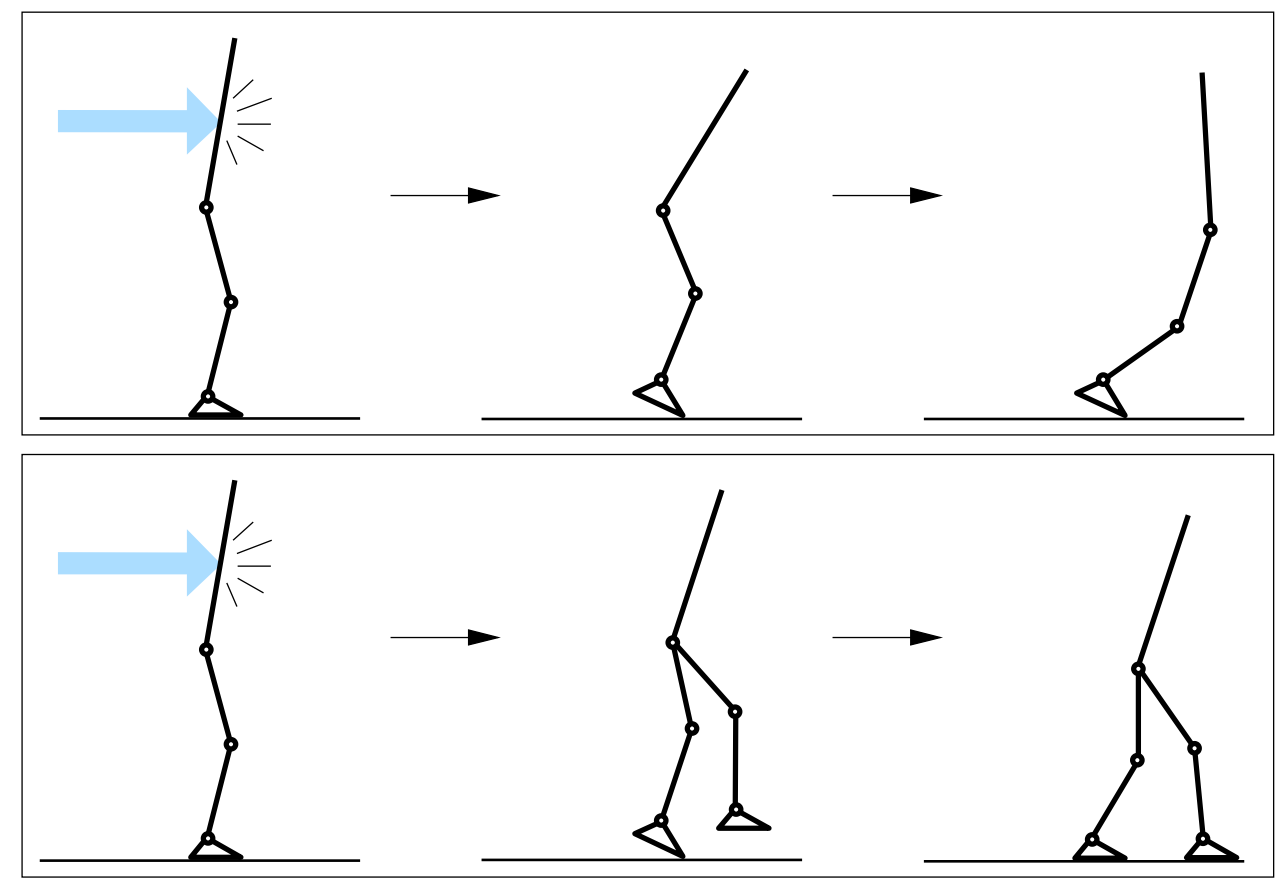

Fig. 2. Tracking a reference trajectory, here a static position, may lead to a fall if the system is outside the corresponding largest invariant set, because of a perturbation for example (upper part). But in such a case, the system might be able to track another trajectory, here a step forward, which would allow to avoid to fall (lower part).

if a trajectory and a neighborhood of it lie inside the viability kernel, it may be tracked with a stable control law, what may allow to avoid to fall.

But whatever the trajectory and the control law, such a tracking will allow to avoid to fall only in a subset of the viability kernel, the largest invariant set for this tracking inside the viability kernel (usually a time-dependent set). This means that if the system is put in a state outside this invariant set, because of a perturbation for example, the tracking will lead to a fall (upper part of figure 2). But in such a case, the state of the system might still be inside the viability kernel, so that there might still be a possibility to avoid to fall.

For example, we may know another reference trajectory for which the state of the system is inside the corresponding largest invariant set, so that tracking this other trajectory would allow to avoid to fall (lower part of figure 2). A possibility then to improve the stability of walking systems could be to adapt the choice of the trajectory being tracked to the actual state of the system. But the problem then is to know when a trajectory can be safely tracked and when not: estimating the extent of the largest invariant set of a trajectory tracking may not be impossible [14], but it is out of reach today.

Our proposition then is to consider stiff enough tracking control laws: requiring 
strong accelerations if a deviation from the reference trajectories occurs, they may satisfy condition (5) only in small neighborhoods around them, but in these neighborhoods, we may trust their ability to stay close enough to the trajectories to avoid that a fall happens. This way, the choice of the reference trajectory to track will be the choice of a trajectory for which such a stiff tracking control law satisfies condition (5).

\subsection{About reference trajectories}

A trajectory can be seen as a set of positions $q_{d}(t)$ indexed with time $t$. But on top of that, we will consider a set of parameters $p$ to describe each possible reference trajectory, for example the step lengths, heights or any other possible variation of the walking patterns, so that to each value of these parameters corresponds a reference trajectory $q_{d}(t, p)$ and reciprocally. This way, a change in the choice of the reference trajectory is perfectly reflected as a variation of these parameters $p$ : for example, instantaneous switches from one reference trajectory to another gives rise to a piecewise constant function $p(t)$.

But because of the second order dynamics (1), such instantaneous switches will most probably give rise to transient behaviors of the trajectory tracking. We will prefer here to take care of such transient behaviors directly in the choice of the reference trajectory: we will consider only continuous variations of the parameters $p(t)$.

More precisely, the second order dynamics will imply that the parameters $p(t)$ be twice differentiable with respect to time, and that on top of being twice differentiable with respect to time (except at impact times), the reference trajectories $q_{d}(t, p)$ be also twice differentiable with respect to the parameters $p$. Of course, this requires that a continuous and twice differentiable set of reference trajectories be at disposal (section 4.2).

Now, any twice differentiable function $p(t)$ can give rise to a trajectory $q_{d}(t, p(t))$ that can be usefully tracked, but which may probably not preserve any specific properties of the original trajectories, those with constant parameters $p$. Examples of such properties not preserved can be the energy optimality of the movements or the fact that the velocity $\dot{q}_{d}(t, p(t))$ may not respect the impact law (6) when $\dot{p}(t) \neq 0$, inducing a flaw in the trajectory tracking at impact times. Such transient behaviors, when $\dot{p}(t) \neq 0$ and $\ddot{p}(t) \neq 0$, should therefore be used thriftily, only when they are strictly necessary to avoid to fall.

We will also be interested in replacing the time $t$ by a virtual time $\tau(t)$, since such a time scaling has proved to be an efficient way to adapt reference trajectories to the dynamical constraints of a system [3]. This way, the parametrization of the reference trajectories $q_{d}(\tau(t), p(t))$ is such that this $\tau(t)$ can be 


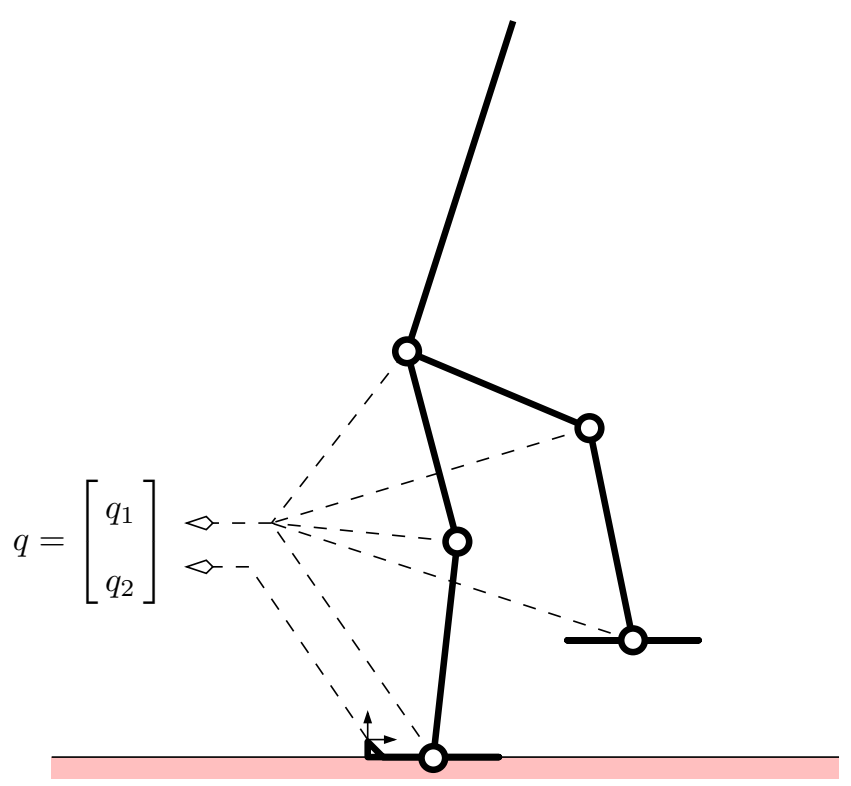

Fig. 3. Structure of the configuration vector $q$ of the 7 link planar biped robot: $q_{1}$ for the joint positions, $q_{2}$ for the position and orientation of a frame attached to the foot on the ground.

directly included in the parameters $p(t)$ : the parametrization of the reference trajectories will therefore appear to be simply $q_{d}(p(t))$.

\section{Application to a simple biped model}

\subsection{A simple biped model}

Let's consider now a 7 link planar biped robot with two feet (of length $22 \mathrm{~cm}$ but with no mass and inertia), two shanks $\left(0.4 \mathrm{~m}, 3.2 \mathrm{~kg}\right.$ and $\left.0.93 \mathrm{~kg} . \mathrm{m}^{2} \mathrm{each}\right)$, two thighs $\left(0.4 \mathrm{~m}, 6.8 \mathrm{~kg}\right.$ and $1.08 \mathrm{~kg} . \mathrm{m}^{2}$ each $)$ and a trunk $(0.625 \mathrm{~m}, 20 \mathrm{~kg}$ and $2.22 \mathrm{~kg} . \mathrm{m}^{2}$ ) for which, following the presentation of section 2.1 , the configuration vector $q$ is structured as: $q_{1}$ for the joint positions (ankles, knees and hips), $q_{2}$ for the position and orientation of a frame attached to the foot on the ground, for example the position of the heel and the angle between the foot and the ground (figure 3). In order to keep this model simple, we consider that both feet are always parallel to the ground, and that an impact occurs when the foot in the air lands on the ground (section 2.3), in such a way that it stays in contact with the ground and the foot previously on the ground takes off immediately: the two feet switch their roles instantly, and we change the model accordingly.

This way, the robot is always in a single support phase except at impact times 
and we define the contact forces in this case as the tangential force, the normal force and the momentum acting under the support foot. $C_{1}(q)$ appears then to be a null matrix, $C_{2}(q)$ an invertible matrix and the inequality (4) a linear inequality:

$$
\mathcal{A}(\lambda)=A \lambda-a \leq 0
$$

stating that the tangential and normal force lie inside the friction cone and that the Centre of Pressure of the contact forces lies within the boundaries of the contact points [14]. We consider also that all the joints are actuated with $T_{1}(q)$ an identity matrix so that the dynamics (2) of the system appears to be in fact:

$$
\begin{aligned}
& M_{1}(q) \ddot{q}+N_{1}(q, \dot{q}) \dot{q}+G_{1}(q)=u \\
& M_{2}(q) \ddot{q}+N_{2}(q, \dot{q}) \dot{q}+G_{2}(q)=C_{2}(q)^{T} \lambda
\end{aligned}
$$

On top of that, the shape and the mechanical behaviour of the ground, and the way we will design the control law in sections 4.3 and 4.4 are such that the foot on the ground will always be considered as motionless on a horizontal ground: $q_{2}$ will be a constant $\Leftrightarrow \dot{q}_{2}=\ddot{q}_{2}=0$. A reduced model is therefore straightforward, with only $q_{1}$ to describe the configuration of the system:

$$
\begin{aligned}
M_{1}^{\prime}\left(q_{1}\right) \ddot{q}_{1}+N_{1}^{\prime}\left(q_{1}, \dot{q}_{1}\right) \dot{q}_{1}+G_{1}^{\prime}\left(q_{1}\right) & =u \\
C_{2}^{-T}\left[M_{2}^{\prime}\left(q_{1}\right) \ddot{q}_{1}+N_{2}^{\prime}\left(q_{1}, \dot{q}_{1}\right) \dot{q}_{1}+G_{2}^{\prime}\left(q_{1}\right)\right] & =\lambda \\
A \lambda & \leq a
\end{aligned}
$$

$\left(C_{2}(q)\right.$ is a constant matrix in this case). Some bounds on the actuation forces are also considered:

$$
B u \leq b
$$

so that combining the dynamics (7)-(8) with the inequalities (9)-(10), a joint movement $q_{1}(t)$ with a motionless foot on the ground can be realized if and only if it complies with the inequality:

$$
\left[\begin{array}{cc}
B & 0 \\
0 & A C_{2}^{-T}
\end{array}\right]\left[M^{\prime}\left(q_{1}\right) \ddot{q}_{1}+N^{\prime}\left(q_{1}, \dot{q}_{1}\right) \dot{q}_{1}+G^{\prime}\left(q_{1}\right)\right] \leq\left[\begin{array}{l}
b \\
a
\end{array}\right]
$$

which is the specific version of condition (5) for this system. A movement that does not satisfy this condition is a movement that cannot be realized as such. The control laws that we are going to consider in the following sections need 


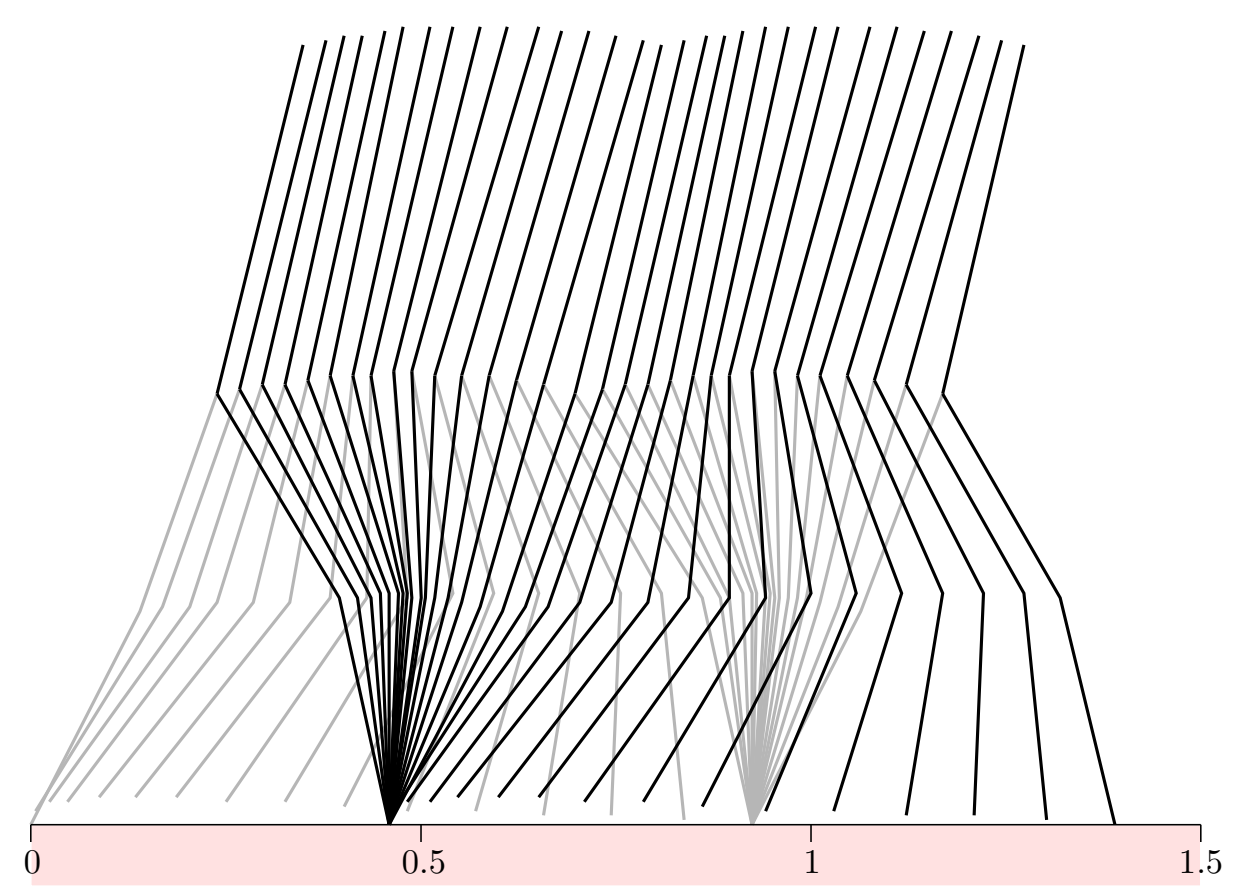

Fig. 4. The $1 m . s^{-1}$ optimal walking motion with instantaneous double support phases due to impacts is obtained with a step length of $0.462 \mathrm{~m}$ (feet not shown).

therefore to comply with this inequality, or they will fail realizing the intended movement, what may potentially lead to undesired outcomes such as falling.

Since satisfying it or not can have a tremendous impact on the safety of both the system and its environment, it can be of importance to consider a robust version of this inequality, allowing for example to take care of modeling errors. A safety margin will be considered therefore by shifting slightly the constants $a$ and $b$ in this inequality to smaller constants $a^{\prime}<a$ and $b^{\prime}<b$. On this specific biped model, we will consider for such a safety margin that the Center of Pressure must always lie at least $1 \mathrm{~cm}$ inside the boundaries of the contact points.

\subsection{The set of reference trajectories}

For this biped robot, we will consider cyclic reference trajectories, with instantaneous double support phases because of the impacts. In order to exploit the strategy of online adaptation proposed in section 3, we will consider a set of trajectories with step lengths varying continuously between 0.3 and $0.65 \mathrm{~m}$, and a time scaling will be applied. Two parameters will be considered then, the step length $p_{1}$ and the virtual time $p_{2}$.

The joint trajectories will be taken between two successive impacts as $4^{\text {th }}$ degree polynomials of the virtual time $p_{2}$, with coefficients being themselves 


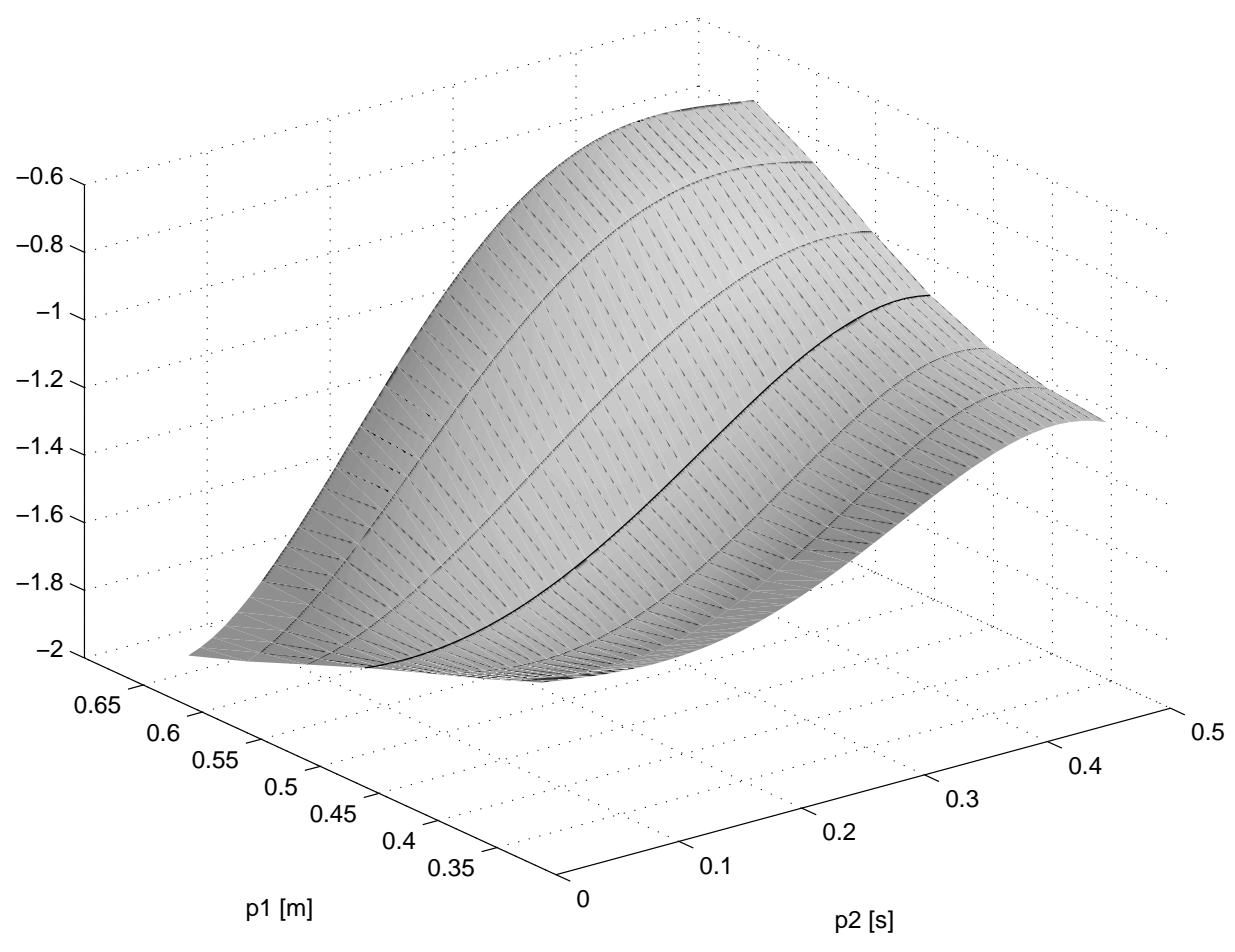

Fig. 5. Evolution of the position (in $[\mathrm{rad}]$ ) of an arbitrary joint with respect to the step length $p_{1}$ varying between 0.3 and $0.65 \mathrm{~m}$ and the virtual time $p_{2}$ varying between 0 and $0.462 s$.

$4^{\text {th }}$ degree polynomials of the step length $p_{1}$ :

$$
q_{1 d}(p)=\sum_{j=0}^{4} \sum_{k=0}^{4}\left(a_{k j} p_{1}^{k}\right) p_{2}^{j}
$$

They are obtained as follows:

- First, a walking motion with an average speed of $1 \mathrm{~m} . \mathrm{s}^{-1}$ is obtained by computing the duration $T$ of the step and the coefficients of a $4^{\text {th }}$ degree polynomial describing the joint trajectory such that the energy consumption $\int_{0}^{T} u^{2} d t$ is minimized, satisfying the continuous dynamics (7)-(10) and the impact law (6). In fact this trajectory is set to satisfy an alternative version of inequality (9): the Center of Pressure is set to stay at least $2 \mathrm{~cm}$ inside the boundaries of the contact points, in order to allow a tolerance margin for the trajectory tracking. This optimal walking motion is obtained with a step length of $0.462 \mathrm{~m}$, realized therefore in $0.462 \mathrm{~s}$ (figure 4), and will be considered as the preferred motion for this system.

- Then, eight other trajectories are computed in a similar way, but for a duration fixed at $0.462 \mathrm{~s}$, and step lengths fixed between 0.3 and $0.65 \mathrm{~m}$ (figure 4 ). Incidentally, we will consider now that the virtual time $p_{2}$ always 


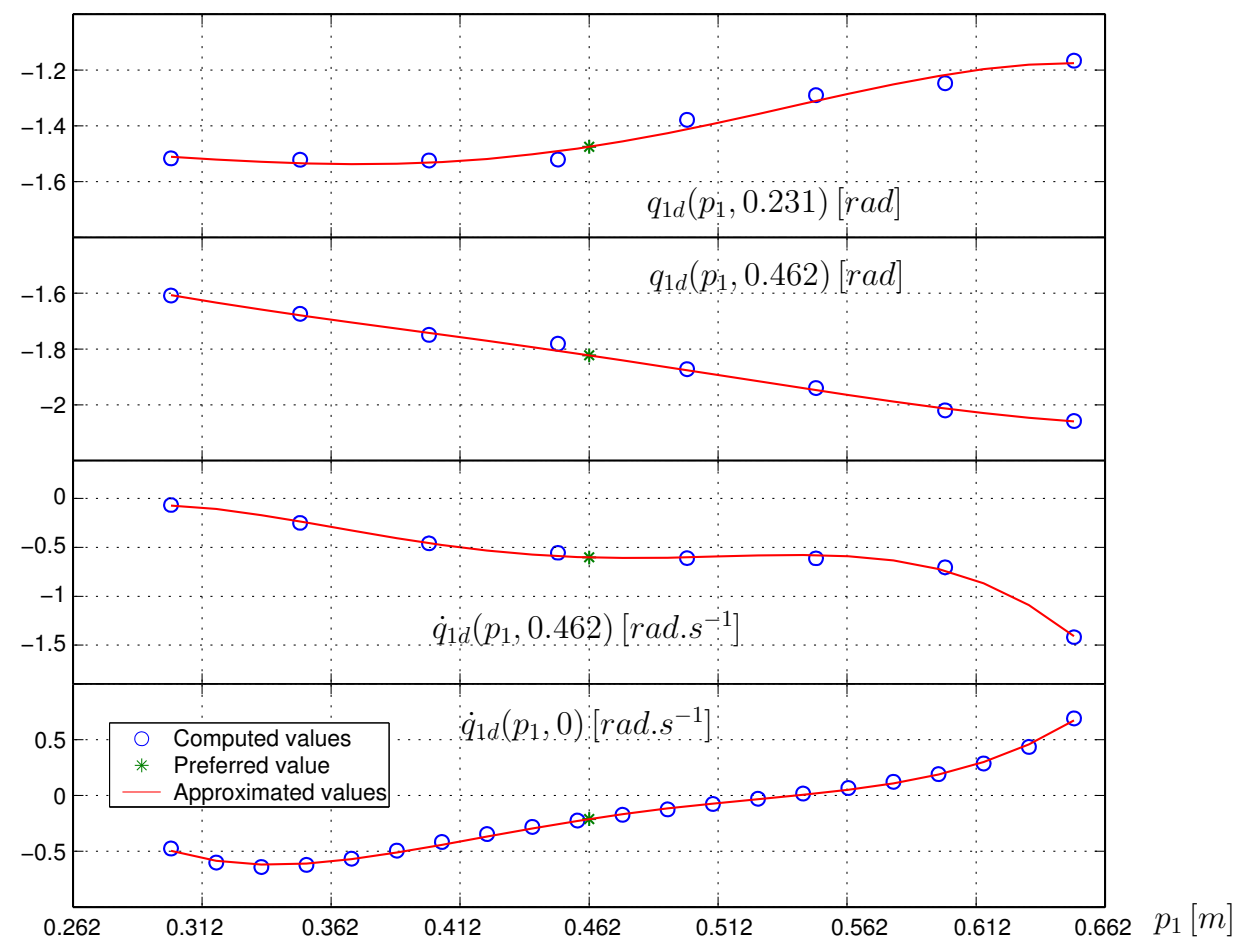

Fig. 6. Result on an arbitrary joint of the $4^{\text {th }}$ degree polynomials of $p_{1}$ interpolating the positions at the middle of the steps $\left(p_{2}=0.231\right)$, at their end $\left(p_{2}=0.462\right)$, and the velocities at their end. The velocities at their beginning $\left(p_{2}=0\right)$ are computed with the impact law for an extensive series of step lengths $p_{1}$, and interpolated only after.

takes values between 0 and 0.462 .

- After that, positions at the middle of the trajectories $\left(p_{2}=0.231\right)$ and positions and velocities at their end $\left(p_{2}=0.462\right)$ are obtained for every step length $p_{1} \in[0.3,0.65]$ by interpolating with $4^{\text {th }}$ degree polynomials of $p_{1}$ the corresponding positions and velocities taken from these nine trajectories (figure 6). Note that this interpolation is specifically computed to match exactly the preferred motion when $p_{1}=0.462$.

- In order to obtain cyclic trajectories when $p_{1}$ is constant, the positions at their beginning directly reproduce the positions at their end, and the velocities at their beginning are computed from the velocities at their end through the impact law (6) for an extensive series of step lengths and interpolated only after (figure 6 ). Remember however that as soon as $\dot{p}_{1} \neq 0$, the velocity $\dot{q}_{d}(p(t))$ will probably not follow the impact law, and the tracking will be flawed.

- In the end, the coefficients of the polynomial (12) can be exactly fit to these positions and velocities already expressed as $4^{\text {th }}$ degree polynomials of $p_{1}$.

Figure 6 shows that in the joint space, the $4^{\text {th }}$ degree polynomials of $p_{1}$ produce an efficient interpolation of the beginning, middle and end positions and 
velocities. But the usual side-effect of such interpolations is an incorrect positioning of the system in the cartesian space: we can observe especially an incorrect height of the feet, inducing a mismatch in the impact times and, as a result, an error in the trajectory tracking, what will be clearly observed in sections 5.1 and 5.2. Note though that the interpolation has been computed to match exactly the preferred motion: this trajectory being unaltered by the interpolation process, no mismatch of the impact times will occur when $p_{1}=0.462$.

\subsection{A trajectory tracking control law}

These reference trajectories will be tracked with a computed torque control law, a feedback linearization:

$$
u=M_{1}^{\prime}\left(q_{1}\right) v+N_{1}^{\prime}\left(q_{1}, \dot{q}_{1}\right) \dot{q}_{1}+G_{1}^{\prime}\left(q_{1}\right)
$$

the result of which on the dynamics (7) is to obtain $\ddot{q}_{1}=v$, that we combine with a PD control law:

$$
v=\ddot{q}_{1 d}(p(t))+K\left(q_{1}-q_{1 d}(p(t))\right)+\Lambda\left(\dot{q}_{1}-\dot{q}_{1 d}(p(t))\right)
$$

Following the proposition of section 3.2, we consider only twice differentiable variations of the parameters $p(t)$ so that the velocity $\dot{q}_{1 d}$ and the acceleration $\ddot{q}_{1 d}$ of the reference trajectory can be related directly to the derivatives of these parameters:

$$
\begin{aligned}
& \dot{q}_{1 d}(p(t))=J(p(t)) \dot{p}(t) \\
& \ddot{q}_{1 d}(p(t))=J(p(t)) \ddot{p}(t)+n(p(t), \dot{p}(t))
\end{aligned}
$$

with $J(p)=\frac{d}{d p} q_{1 d}(p)$ and $n(p, \dot{p})=\left(\frac{d}{d t} J(p)\right) \dot{p}$. This way, the PD control law appears as:

$$
v=J(p(t)) \ddot{p}(t)+n(p(t), \dot{p}(t))+K\left(q_{1}-q_{1 d}(p(t))\right)+\Lambda\left(\dot{q}_{1}-J(p(t)) \dot{p}(t)\right)
$$

or, with a shortened notation:

$$
v=J \ddot{p}+n+K\left(q_{1}-q_{1 d}\right)+\Lambda\left(\dot{q}_{1}-J \dot{p}\right)
$$


In order to improve the capacity for the robot to avoid to fall, following the analysis of section 3.1, the choice of the reference trajectory to track should be made according to the state of the system. More precisely, the choice should be made of a trajectory for which the control law (13)-(14) complies with the inequality (11), taking into account the safety margin described in the end of section 4.1, satisfying therefore the inequality (with a shortened notation):

$$
\left[\begin{array}{cc}
B & 0 \\
0 & A C_{2}^{-T}
\end{array}\right]\left[M^{\prime}\left(J \ddot{p}+n+K\left(q_{1}-q_{1 d}\right)+\Lambda\left(\dot{q}_{1}-J \dot{p}\right)\right)+N^{\prime} \dot{q}_{1}+G^{\prime}\right] \leq\left[\begin{array}{l}
b^{\prime} \\
a^{\prime}
\end{array}\right]
$$

Taking care of choosing a reference trajectory which complies with this inequality, we would like now to have the robot stay as close as possible to an optimal motion, a $1 \mathrm{~m} . \mathrm{s}^{-1}$ walk obtained with $p_{1}(t)=0.462$ and $\dot{p}_{2}(t)=1$ (see section 4.2 and figure 4), following it if possible and getting back to it if driven away by a perturbation. Such a behaviour can be obtained with a PD regulation of the walking parameters:

$$
\ddot{p}_{\times}=\left[\begin{array}{c}
k_{1}\left(p_{1}-0.462\right)+k_{2} \dot{p}_{1} \\
k_{3}\left(\dot{p}_{2}-1\right)
\end{array}\right]
$$

but having to comply with the inequality (15), we rather need to consider the following evolution law:

$$
\ddot{p}=\arg \min _{\pi}\left\|\pi-\ddot{p}_{\times}\right\|
$$

s.t. the inequality (15) is satisfied.

Indeed, trying to stay as close as possible to the optimal motion, this evolution law explicitly selects a reference trajectory for which the control law (13)-(14) complies with the inequality (11) (note that we regulate the pace of the steps $\dot{p}_{2}$ without synchronization between the virtual time $p_{2}$ and the real time $t$ ).

Here appears a regulation of the parameters $p_{1}$ and $p_{2}$ : note that these are parameters of the control law that exist only inside of the control law and don't need therefore to be measured in the physical world. The dynamics of these parameters is purely abstract.

General Remark : In a way, tracking a single reference trajectory $q_{d}(t)$ imposes an unnecessary and unproductive reduction of the walking behaviour: 
the two-step control law that we propose here, (13)-(14) together with (16)(17), can be seen therefore as a way to widen this walking behaviour to a set $q_{d}(p)$ with $\operatorname{dim}(p)>1$, giving back some freedom of movement to the walking system to help it cope with the dynamical constraints (11) and adapt to its environment. Indeed, a single tracking control such as (13)-(14) mobilizes all the actuators of the system to control the positions and velocities of each of its articulations, leaving no simple possibility to deal then with such constraints. The term $\ddot{p}$ that appears in (14) is used then in our scheme as a new input to the control law (13)-(14) which is used in (16)-(17) to deal explicitly with such constraints, and once this is done, to deal with more specific goals if it is possible.

\section{Simulations}

\subsection{Perturbation of a trajectory tracking}

Let's consider this simple biped model perfectly tracking the $1 \mathrm{~m} . \mathrm{s}^{-1}$ optimal walk $\left(p_{1}=0.462, \dot{p}_{2}=1\right)$ when a perturbation occurs, a horizontal force applied on the back of the trunk for $0.025 \mathrm{~s}$ at the middle of a step. This perturbation might put the system in a state outside the largest invariant set corresponding to this trajectory tracking, so a change of reference trajectory might be necessary to avoid to fall.

When no adaptation of the reference trajectory is applied, external forces as large as $55 N$ can be compensated by the control law (13)-(14) before it stops complying with the inequality (11). When the strategy that we have proposed is applied, but adapting only the parameter $p_{1}$, external forces as large as $235 N$ can be compensated. When only the parameter $\dot{p}_{2}$ is adapted, external forces as large as $450 N$ can be compensated. And when both parameters are adapted, external forces as large as $750 \mathrm{~N}$ can be compensated: this strategy clearly improves the capacity for this biped model to avoid to fall.

Figure 7 shows the application of our strategy when a $600 N$ force is applied: a strong rise of the tracking error is induced, but the strategy reacts immediately by increasing slightly the step length $(5 \mathrm{~mm})$ and mostly by increasing its pace (40\% faster). A step later, the system has completely come back to the original $1 \mathrm{~m} . \mathrm{s}^{-1}$ optimal walk, and falling has been avoided.

Note that there is a sudden rise of the tracking error at the first impact time: the tracking may be flawed because $\dot{p}_{1} \neq 0$, inducing a velocity $\dot{q}_{1 d}$ which doesn't follow the impact law (6), but it seems to be mostly flawed because $p_{1} \neq 0.462$, inducing a mismatch in the impact times because of the 


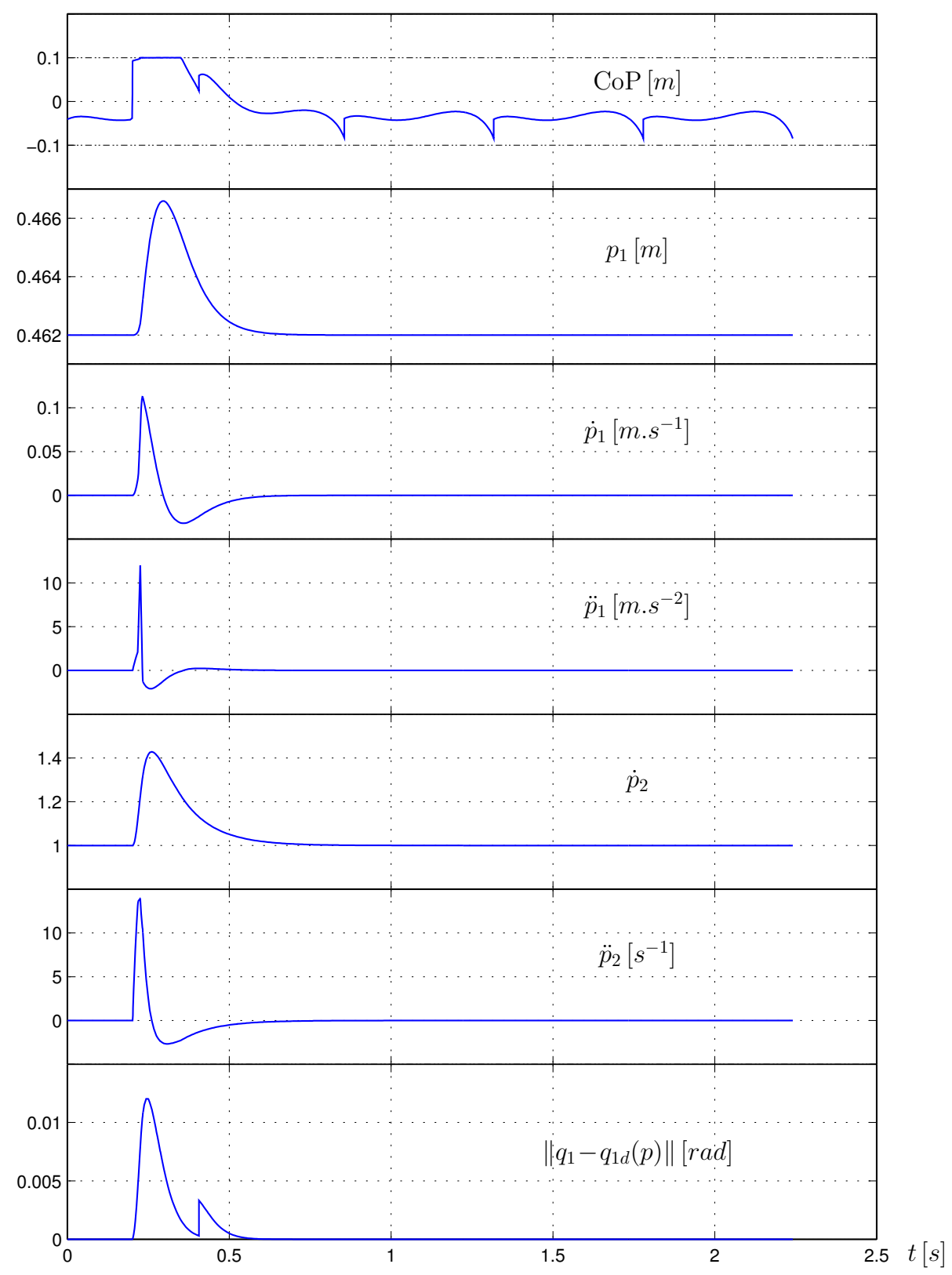

Fig. 7. Position of the Center of Pressure within the bounds of the safety margin under the foot on the ground, variation of the walking parameters $p_{1}$ and $\dot{p}_{2}$ and tracking error when a $600 N$ external force is applied on the back of the system for $0.025 \mathrm{~s}$.

interpolation realized in section 4.2 .

\subsection{Avoiding a hole}

Consider again this walking system tracking perfectly the $1 \mathrm{~m} . \mathrm{s}^{-1}$ optimal walk $\left(p_{1}=0.462, \dot{p}_{2}=1\right)$ when a sensor such as a vision sensor suddenly 


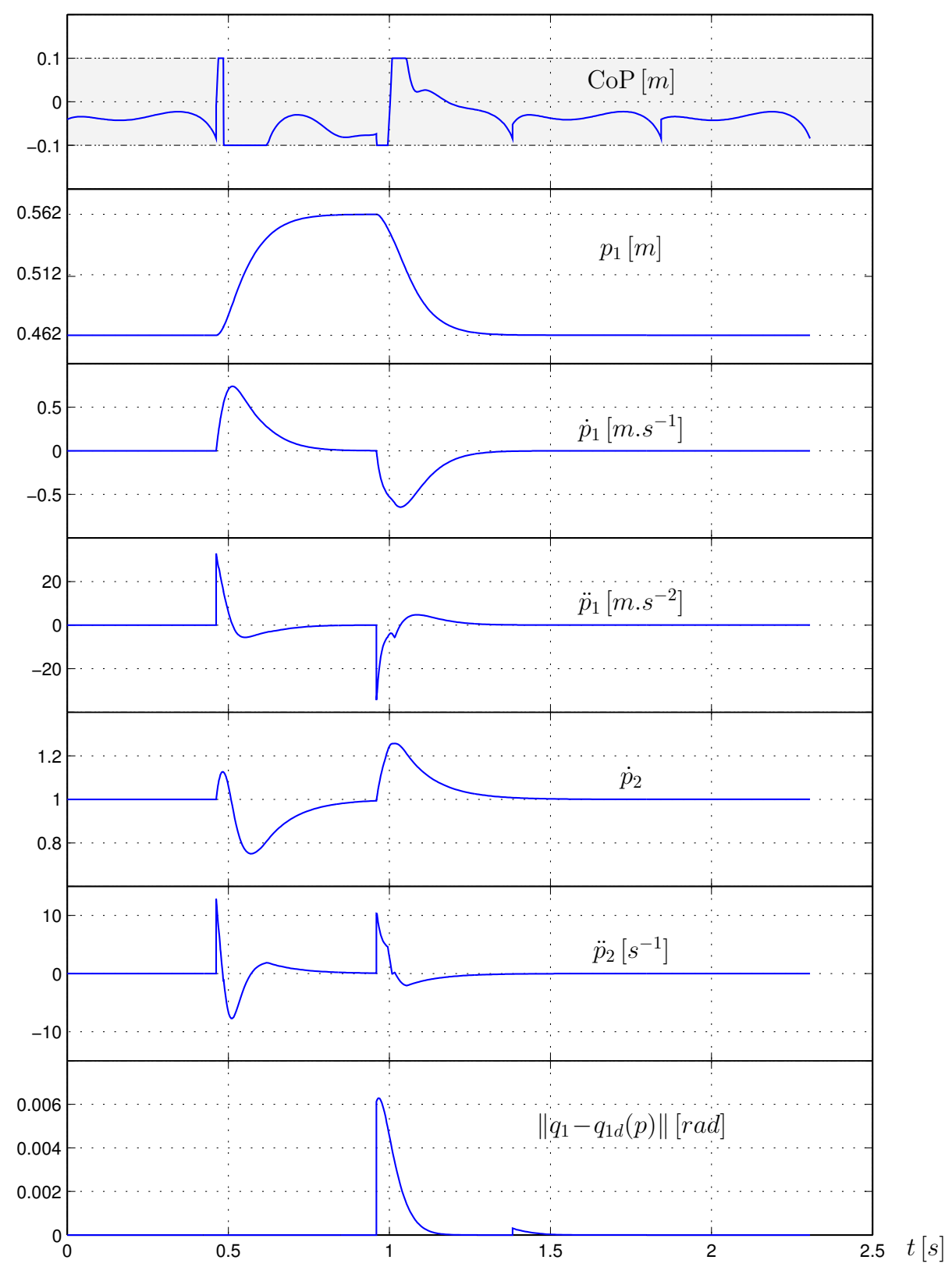

Fig. 8. Position of the Center of Pressure within the bounds of the safety margin under the foot on the ground, variation of the walking parameters $p_{1}$ and $\dot{p}_{2}$ and tracking error when a hole has to be avoided at the last moment.

detects, at the beginning of a step, that this step should be $10 \mathrm{~cm}$ longer in order to avoid a hole ( $p_{1}$ should be 0.562$)$.

One strategy could be to switch instantaneously the parameter $p_{1}$ to the required value, but because of the stiff transient behaviour of the trajectory tracking that such a switch induces, subsequent adaptations of both $p_{1}$ and $\dot{p}_{2}$ would be required to avoid to fall. 
We will prefer therefore to stick to the proposition of section 3.2 of taking care of the transient behaviour directly in the choice of the reference trajectory: we will simply switch the reference value in (16) from 0.462 to 0.562 , and we can see on figure 8 that this induces a nice exponential convergence of the step length, with virtually no tracking error. Once again, there is a sudden rise of the tracking error at each impact time where $p_{1} \neq 0.462$, but it is because of the mismatch in the impact times induced by the interpolation realized in section 4.2 .

This example demonstrates the possibility to integrate to the proposed control scheme a higher-level supervisor which would drive the choice of the reference trajectory according to informations gathered on the environment of the walking system.

\section{Conclusion}

We have seen that the movements of walking systems and therefore their capacity to avoid to fall are bound to the availability of contact forces. A classical way to deal with these available contact forces is to track reference trajectories that have been carefully designed to deal properly with them, but this usually offers only a limited amount of stability: even small perturbations of the tracking may lead to a fall.

Many propositions have been made then to improve this stability, all heuristics that keep an eye on the available contact forces and either lower the objectives of the trajectory tracking or adapt them when required. Our proposition here is to always fully track a trajectory that has been designed and fixed in advance, but to continuously adapt the choice of the reference trajectory being tracked to the available contact forces.

This general heuristic should be widely applicable to different walking systems, planar or three-dimensional, with any number of legs with or without feet, but we have chosen for the sake of simplicity to show here how it can be applied to a simple planar biped model, with very promising simulation results. To apply this heuristic more generally should barely require a basic tracking control law for the walking system being considered, dealing for example with multiple support phases or under-actuation, and a set of reference trajectories that follows the few rules described in section 3. All this is already available for example for fully three-dimensional humanoid robots $[5,10]$. 


\section{References}

[1] J.-P. Aubin. Viability Theory. Birkhäuser, 1991.

[2] C. Azevedo, P. Poignet, and B. Espiau. On line optimal control for biped robots. In Proceedings of the IFAC $15^{\text {th }}$ World Congress, 2002.

[3] C. Chevallereau. Time scaling control for an underactuated biped robot. IEEE Trans. on Robotcs and Automation, 19(2):362-368, 2003.

[4] C. Chevallereau and B. Perrin. Computed torque control law under constraints for single and double support motion of planar biped robot. In Proceedings of ECPD'98 : european Center for Peace and Development, 1998.

[5] J. Denk and G. Schmidt. Synthesis of walking primitive databases for biped robots in 3d environments. In Proceedings of the IEEE International Conference on Robotics \& Automation, 2003.

[6] Y. Fujimoto, S. Obata, and A. Kawamura. Robust biped walking with active interaction control between foot and ground. In Proceedings of the IEEE International Conference on Robotics \&6 Automation, 1998.

[7] F. Génot and B. Espiau. On the control of the mass center of legged robots under unilateral constraints. In Proceedings of the International Symposium on Mobile, Climbing and Walking Robots, 1998.

[8] K. Hirai, M. Hirose, Y. Haikawa, and T. Takenaka. The development of honda humanoid robot. In Proceedings of the IEEE International Conference on Robotics \& Automation, 1998.

[9] Y. Hurmuzlu, F. Génot, and B. Brogliato. Modeling, stability and control of biped robots: A general framework. INRIA research report 4290, 2001.

[10] K. Löffler, M. Gienger, and F. Pfeiffer. Sensor and control design of a dynamically stable biped robot. In Proceedings of the IEEE International Conference on Robotics \& Automation, 2003.

[11] J. Ostrowski and J. Burdick. Geometric perspectives on the mechanics and control of robotic locomotion. In Proceedings of the International Symposium on Robotics Research, 1995.

[12] M. Vukobratovic, B. Borovac, D. Surla, and D. Stokic. Biped locomotion. Springer-Verlag, Berlin, 1990.

[13] P.B. Wieber. Modélisation et commande d'un robot marcheur anthropomorphe. $\mathrm{PhD}$ thesis, Mines de Paris, 2000.

[14] P.B. Wieber. On the stability of walking systems. In Proceedings of the International Workshop on Humanoid and Human Friendly Robotics, 2002.

[15] K. Yokoi, F. Kanchiro, K. Fujiwara, S. Kajita, and H. Hirokawa. Experimental study of biped locomotion of humanoid robot hrp-1s. In Procedings of the 8th International Symposium on Experimental Robotics ISER'02, 2002. 